\title{
Set-Valued Hardy-Rogers Type Contraction in 0-Complete Partial Metric Spaces
}

\author{
Satish Shukla, ${ }^{1}$ Stojan Radenović, ${ }^{2}$ and Calogero Vetro ${ }^{3}$ \\ ${ }^{1}$ Department of Applied Mathematics, Shri Vaishnav Institute of Technology \& Science, Gram Baroli, Sanwer Road, \\ Indore 453331, India \\ ${ }^{2}$ Faculty of Mechanical Engineering, University of Belgrade, Kraljice Marije 16, 11120 Belgrade, Serbia \\ ${ }^{3}$ Dipartimento di Matematica e Informatica, Università degli Studi di Palermo, Via archirafi 34, 90123 Palermo, Italy \\ Correspondence should be addressed to Satish Shukla; satishmathematics@yahoo.co.in
}

Received 27 January 2014; Accepted 19 March 2014; Published 8 April 2014

Academic Editor: Hernando Quevedo

Copyright (C) 2014 Satish Shukla et al. This is an open access article distributed under the Creative Commons Attribution License, which permits unrestricted use, distribution, and reproduction in any medium, provided the original work is properly cited.

In this paper we introduce set-valued Hardy-Rogers type contraction in 0-complete partial metric spaces and prove the corresponding theorem of fixed point. Our results generalize, extend, and unify several known results, in particular the recent Nadler's fixed point theorem in the context of complete partial metric spaces established by Aydi et al. (2012). As an application of our results, a homotopy theorem for such mappings is derived. Also, some examples are included which show that our generalization is proper.

\section{Introduction and Preliminaries}

The well-known Banach contraction mapping principle states that, if $(X, d)$ is a complete metric space and $T: X \rightarrow X$ is a self-mapping such that

$$
d(T x, T y) \leq \lambda d(x, y)
$$

for all $x, y \in X$, where $\lambda \in[0,1)$, then $f$ has a fixed point in $X$. Because of simplicity and several applications, Banach principle was generalized by several authors, in different directions.

For instance, Hardy and Rogers [1] used the contractive condition

$$
\begin{aligned}
d(T x, T y) \leq & a_{1} d(x, y)+a_{2} d(x, T x) \\
& +a_{3} d(y, T y)+a_{4} d(x, T y)+a_{5} d(y, T x)
\end{aligned}
$$

for all $x, y \in X$, where $a_{1}, a_{2}, a_{3}, a_{4}, a_{5}$ are nonnegative constants such that $\sum_{i=1}^{5} a_{i}<1$, and proved fixed point result. Note that condition (2) generalizes the contractive conditions of Banach, Kannan, Reich, Chatterjea, and Ćirić (see [2]).
It is well known that the theory of set-valued mappings has application in control theory, convex optimization, differential equations, and economics. Nadler Jr. [3] generalized the Banach contraction mapping principle to set-valued mappings and proved the following fixed point theorem.

Theorem 1. Let $(X, d)$ be a complete metric space and let $T$ be a mapping from $X$ into $C B(X)$ (here $C B(X)$ denotes the set of all nonempty closed bounded subset of $X)$ such that, for all $x, y \in X$,

$$
H(T x, T y) \leq \lambda d(x, y)
$$

where $\lambda \in[0,1)$. Then $T$ has a fixed point.

In recent years, Matthews [4] introduced the notion of partial metric space as a part of the study of denotational semantics of dataflow networks, with the interesting property of "nonzero self-distance" in space. He showed that the Banach contraction mapping principle can be generalized to the partial metric context for applications in program verification. For a more detailed explanation we refer the reader to Bukatin et al. [5] where the motivation for introducing nonzero distance is explained, which is also leading 
to interesting research in foundations of topology. Later on, Romaguera [6] introduced the notions of 0-Cauchy sequence and 0 -complete partial metric spaces and proved some characterizations of partial metric spaces in terms of completeness and 0-completeness.

Very recently, Aydi et al. [7] introduced the notion of partial Hausdorff metric and extended the Nadler's theorem in partial metric spaces. In this paper, we discuss some properties of partial metric spaces and extend the results of Kadelburg et al. [8], Altun et al. [9], and Aydi et al. [7] for setvalued mappings in 0 -complete partial metric spaces. As an application, we prove a homotopy result which extends and generalizes the homotopy result of $[7,9]$ for set-valued mappings in 0 -complete partial metric spaces.

Consistent with $[4,6-10]$, the following definitions and results will be needed in the sequel.

Definition 2 (see [4]). A partial metric on a nonempty set $X$ is a function $p: X \times X \rightarrow \mathbb{R}^{+}\left(\mathbb{R}^{+}\right.$stands for the set of nonnegative reals) such that, for all $x, y, z \in X$,

$$
\begin{aligned}
& \text { (p1) } x=y \Leftrightarrow p(x, x)=p(x, y)=p(y, y), \\
& \text { (p2) } p(x, x) \leq p(x, y) \\
& \text { (p3) } p(x, y)=p(y, x) \\
& \text { (p4) } p(x, y) \leq p(x, z)+p(z, y)-p(z, z) .
\end{aligned}
$$

A partial metric space is a pair $(X, p)$ such that $X$ is a nonempty set and $p$ is a partial metric on $X$.

It is clear that, if $p(x, y)=0$, then from (P1) and (P2) $x=y$. But, if $x=y, p(x, y)$ may not be 0 . Also every metric space is a partial metric space, with zero self-distance.

Example 3. If $p: \mathbb{R}^{+} \times \mathbb{R}^{+} \rightarrow \mathbb{R}^{+}$is defined by $p(x, y)=$ $\max \{x, y\}$, for all $x, y \in \mathbb{R}^{+}$, then $\left(\mathbb{R}^{+}, p\right)$ is a partial metric space.

Some more examples of partial metric spaces can be seen in $[4,7,8]$.

Each partial metric on $X$ generates a $T_{0}$ topology $\tau_{p}$ on $X$ which has as a base the family of open $p$-balls $\left\{B_{p}(x, \epsilon): x \epsilon\right.$ $X, \epsilon>0\}$, where $B_{p}(x, \epsilon)=\{y \in X: p(x, y)<p(x, x)+\epsilon\}$ for all $x \in X$ and $\epsilon>0$.

Theorem 4 (see [4]). For each partial metric $p: X \times X \rightarrow$ $\mathbb{R}^{+}$the pair $\left(X, p^{s}\right)$ where $p^{s}(x, y)=2 p(x, y)-p(x, x)-$ $p(y, y)$ for all $x, y \in X$, is a metric space.

Here $\left(X, p^{s}\right)$ is called induced metric space and $p^{s}$ is called induced metric.

Definition 5 (see $[4,9])$. Let $(X, p)$ be a partial metric space. Then,

(1) a sequence $\left\{x_{n}\right\}$ in $(X, p)$ converges to a point $x \in X$ if and only if $p(x, x)=\lim _{n \rightarrow \infty} p\left(x_{n}, x\right)$;

(2) a sequence $\left\{x_{n}\right\}$ in $(X, p)$ is called Cauchy sequence if there exists (and is finite) $\lim _{n, m \rightarrow \infty} p\left(x_{n}, x_{m}\right)$;
(3) $(X, p)$ is said to be complete if every Cauchy sequence $\left\{x_{n}\right\}$ in $X$ converges with respect to $\tau_{p}$ to a point $x \in X$ such that $p(x, x)=\lim _{n, m \rightarrow \infty} p\left(x_{n}, x_{m}\right)$;

(4) a sequence $\left\{x_{n}\right\}$ in $(X, p)$ is called 0 -Cauchy sequence if $\lim _{n, m \rightarrow \infty} p\left(x_{n}, x_{m}\right)=0$. The space $(X, p)$ is said to be 0 -complete if every 0 -Cauchy sequence in $X$ converges with respect to $\tau_{p}$ to a point $x \in X$ such that $p(x, x)=0$.

Lemma 6 (see $[4,6,10])$. Let $(X, p)$ be a partial metric space and $\left\{x_{n}\right\}$ be any sequence in $X$. Then,

(i) $\left\{x_{n}\right\}$ is a Cauchy sequence in $(X, p)$ if and only if it is a Cauchy sequence in $\left(X, p^{s}\right)$;

(ii) $(X, p)$ is complete if and only if the metric space $\left(X, p^{s}\right)$ is complete. Furthermore, $\lim _{n \rightarrow \infty} p^{s}\left(x_{n}, x\right)=$ 0 if and only if $p(x, x)=\lim _{n \rightarrow \infty} p\left(x_{n}, x\right)=$ $\lim _{n, m \rightarrow \infty} p\left(x_{n}, x_{m}\right)$;

(iii) every 0 -Cauchy sequence in $(X, p)$ is Cauchy in $\left(X, p^{s}\right)$;

(iv) if $(X, p)$ is complete, then it is 0 -complete.

The converse assertions of (iii) and (iv) do not hold. Indeed the partial metric space $\left(\mathbb{Q} \cap \mathbb{R}^{+}, p\right)$, where $\mathbb{Q}$ denotes the set of rational numbers and the partial metric $p$ is given by $p(x, y)=\max \{x, y\}$ provides an easy example of a 0 -complete partial metric space which is not complete. It is easy to see that every closed subset of a 0 -complete partial metric space is 0 -complete.

Consistent with [7], we recall the notions of closedness and boundedness in a partial metric space $(X, p)$. Let $C B^{p}(X)$ be the family of all nonempty, closed, and bounded subsets of $(X, p)$, induced by the partial metric $p$. Note that closedness is taken from $\left(X, \tau_{p}\right)\left(\tau_{p}\right.$ is the topology induced by $\left.p\right)$ and boundedness is given as follows: $A$ is a bounded subset in $(X, p)$ if there exist $x_{0} \in X$ and $M \geq 0$ such that, for all $a \in A$, we have $a \in B_{p}\left(x_{0}, M\right)$, that is, $p\left(x_{0}, a\right)<p\left(x_{0}, x_{0}\right)+M$.

Now, for $A, B \in C B^{p}(X)$ and $x \in X$, define

$$
\begin{aligned}
p(x, A) & =\inf \{p(x, a): a \in A\} ; \\
\delta_{p}(A, B) & =\sup \{p(a, B): a \in A\} .
\end{aligned}
$$

Lemma 7 (see [9]). Let $(X, p)$ be a partial metric space and $A$ be any nonempty set in $(X, p)$. Then $a \in \bar{A}$ if and only if $p(a, A)=p(a, a)$, where $\bar{A}$ denotes the closure of $A$ with respect to the partial metric $p$. Note that $A$ is closed in $(X, p)$ if and only if $A=\bar{A}$.

Proposition 8 (see [7]). Let $(X, p)$ be a partial metric space. For all $A, B, C \in C B^{p}(X)$, we have the following:

(i) $\delta_{p}(A, A)=\sup \{p(a, a): a \in A\}$;

(ii) $\delta_{p}(A, A) \leq \delta_{p}(A, B)$;

(iii) $\delta_{p}(A, B)=0$ implies that $A \subseteq B$;

(iv) $\delta_{p}(A, B) \leq \delta_{p}(A, C)+\delta_{p}(C, B)-\inf _{c \in C} p(c, c)$. define

Let $(X, p)$ be a partial metric space. For $A, B \in C B^{p}(X)$,

$$
H_{p}(A, B)=\max \left\{\delta_{p}(A, B), \delta_{p}(B, A)\right\} .
$$


Proposition 9 (see [7]). Let $(X, p)$ be partial metric spaces. For all $A, B, C \in C B^{p}(X)$, we have

(h1) $H_{p}(A, A) \leq H_{p}(A, B)$;

(h2) $H_{p}(A, B)=H_{p}(B, A)$;

(h3) $H_{p}(A, B) \leq H_{p}(A, C)+H_{p}(C, B)-\inf _{c \in C} p(c, c)$.

(h4) $H_{p}(A, B)=0$ implies that $A=B$.

In view of Proposition 9, we call the mapping $H_{p}: C B^{p}$ $(X) \times C B^{p}(X) \rightarrow \mathbb{R}^{+}$a partial Hausdorff metric induced by $p$. result.

The following Lemma is crucial for the proof of our main

Lemma 10. Let $(X, p)$ be a partial metric space, $\epsilon>0$ be arbitrary, and $A, B \in C B^{P}(X)$. For any $a \in A$, there exists $b=$ $b(a) \in B$ such that

$$
p(a, b) \leq H_{p}(A, B)+\epsilon .
$$

Proof. If $A=B$, then, from (i) of Proposition 8, we have

$$
H_{p}(A, B)=H_{p}(A, A)=\delta_{p}(A, A)=\sup _{x \in A} p(x, x) .
$$

Since $A \in C B^{p}(X)$, for $a \in A$, we have

$$
p(a, a) \leq \sup _{x \in A} p(x, x)=H_{p}(A, B)<H_{p}(A, B)+\epsilon .
$$

Therefore the result holds true with $b=a$. If $A \neq B$, suppose there exists $a \in A$ such that for all $b \in B$ we have $p(a, b)>$ $H_{p}(A, B)+\epsilon$. Therefore $\inf \{p(a, y): y \in B\} \geq H_{p}(A, B)+\epsilon$ or $p(a, B) \geq H_{p}(A, B)+\epsilon$. Note that

$$
\begin{aligned}
H_{p}(A, B) & \geq \delta_{p}(A, B) \\
& =\sup _{x \in A} p(x, B) \geq p(a, B) \geq H_{p}(A, B)+\epsilon .
\end{aligned}
$$

As $\epsilon>0$ is arbitrary, we get a contradiction and the result holds true.

Remark 11. Each partial metric on $X$ generates a $T_{0}$ topology $\tau_{p}$ on $X$, but it is not necessarily $T_{1}$, in the sense that a singleton set in partial metric spaces may not be closed. Consider the partial metric space as in Example 3. Let $x \in X$; then the singleton set $\{x\}$ is not a closed set with respect to $p$. Indeed, for any $y \in X$,

$$
\begin{aligned}
y \in \overline{\{x\}} & \Longleftrightarrow p(y,\{x\})=p(y, y) \\
& \Longleftrightarrow \max \{x, y\}=\max \{y, y\} \\
& \Longleftrightarrow y \geq x .
\end{aligned}
$$

Therefore, for any $y>x$, we have $y \in \overline{\{x\}}$ and hence $\{x\}$ is not closed with respect to $p$.

\section{Main Results}

Firstly, we consider some properties of partial metric spaces which will be useful in further discussion.

The following proposition shows that, for every partial metric space $(X, p)$, there exists a partial metric space $\left(X, p^{*}\right)$ such that the topological space $\left(X, \tau_{p^{*}}\right)$ is a Hausdorff space.

Proposition 12. Let $X$ be any nonempty set and $d$ and $p$ be, respectively, a metric and a partial metric on $X$. Define $p^{*}$ : $X \times X \rightarrow \mathbb{R}^{+}$by $p^{*}(x, y)=d(x, y)+p(x, y)$ for all $x, y \in X$. Then $\left(X, p^{*}\right)$ is a partial metric space such that the topological space $\left(X, \tau_{p^{*}}\right)$ is a Hausdorff space.

Proof. Let $x, y \in X$ with $x \neq y$. We shall show that $x$ and $y$ are contained into two disjoint open sets with respect to $p^{*}$. Since $x \neq y$, we have $d(x, y)>0$. Now, let $\epsilon=d(x, y) / 2$; then $B_{p^{*}}(x, \epsilon), B_{p^{*}}(y, \epsilon) \in \tau_{p^{*}}, x \in B_{p^{*}}(x, \epsilon)$, and $y \in B_{p^{*}}(y, \epsilon)$. If $B_{p^{*}}(x, \epsilon) \cap B_{p^{*}}(y, \epsilon) \neq \emptyset$, let $z \in B_{p^{*}}(x, \epsilon) \cap B_{p^{*}}(y, \epsilon)$. Note that

$$
p^{*}(z, x)<\epsilon+p^{*}(x, x), \quad p^{*}(z, y)<\epsilon+p^{*}(y, y) ;
$$

that is

$$
\begin{aligned}
& d(z, x)+p(z, x)<\epsilon+p(x, x), \\
& d(z, y)+p(z, y)<\epsilon+p(y, y) .
\end{aligned}
$$

Using (12) we obtain

$$
\begin{aligned}
d(x, y) & \leq d(x, z)+d(z, y) \\
& <\epsilon+p(x, x)-p(z, x)+\epsilon+p(y, y)-p(z, y) \\
& =2 \epsilon+p(x, x)+p(y, y)-p(z, x)-p(z, y) \\
& =d(x, y)+p(x, x)+p(y, y)-p(z, x)-p(z, y)
\end{aligned}
$$

that is

$$
p(z, x)+p(z, y)<p(x, x)+p(y, y),
$$

a contradiction (as $p(x, x) \leq p(z, x)$ and $p(y, y) \leq p(z, y))$. Therefore, $B_{p^{*}}(x, \epsilon) \cap B_{p^{*}}(y, \epsilon)=\emptyset$ and hence $\left(X, \tau_{p^{*}}\right)$ is a Hausdorff space.

Corollary 13. Let $(X, p)$ be a partial metric space; then there exists a partial metric $p^{*}$ on $X$ such that the topological space $\left(X, \tau_{p^{*}}\right)$ is a Hausdorff space.

Proof. Consider the induced metric $p^{s}$ by $p$ and define $p^{*}(x, y)=p^{s}(x, y)+p(x, y)$ for all $x, y \in X$. By Proposition $12, p^{*}$ is the required partial metric.

Corollary 14. Let $(X, p)$ be a partial metric space; then there exists a partial metric $p^{*}$ on $X$ such that the topological space $\left(X, \tau_{p^{*}}\right)$ is a $T_{1}$ space and therefore every singleton subset of $X$ is closed with respect to $p^{*}$. 
Now we state the fixed point theorem for set-valued Hardy-Rogers type mappings.

Theorem 15. Let $(X, p)$ be a 0 -complete partial metric space and $f: X \rightarrow C B^{p}(X)$ be a mapping such that

$$
\begin{aligned}
H_{p}(f x, f y) \leq & a_{1} p(x, y)+a_{2} p(x, f x) \\
& +a_{3} p(y, f y)+a_{4} p(x, f y)+a_{5} p(y, f x),
\end{aligned}
$$

for all $x, y \in X$, where $a_{1}, a_{2}, a_{3}, a_{4}, a_{5}$ are nonnegative constants with $\sum_{i=1}^{5} a_{i}<1$. Then $f$ has a fixed point in $X$.

Proof. Let $x_{0} \in X$ be arbitrary. We construct a sequence $\left\{x_{n}\right\}$ in $X$ as follows: since $f x_{0} \in C B^{p}(X)$, let $x_{1} \in f x_{0}$. Also, since $f x_{1} \in C B^{P}(X)$, by Lemma 10 , there exists $x_{2} \in f x_{1}$ such that

$$
p\left(x_{1}, x_{2}\right) \leq H_{p}\left(f x_{0}, f x_{1}\right)+\epsilon,
$$

where $\epsilon>0$ is arbitrary. Similarly there exists $x_{3} \in f x_{2}$ such that

$$
p\left(x_{2}, x_{3}\right) \leq H_{p}\left(f x_{1}, f x_{2}\right)+\epsilon^{2} .
$$

Continuing this procedure we construct a sequence $\left\{x_{n}\right\}$ in $X$ such that $x_{n+1} \in f x_{n}$ and

$$
p\left(x_{n}, x_{n+1}\right) \leq H_{p}\left(f x_{n-1}, f x_{n}\right)+\epsilon^{n} \quad \forall n \in \mathbb{N} .
$$

Now, if $x_{n+1}=x_{n}$ for any $n$, then the proof is finished. Therefore, let $x_{n} \neq x_{n+1}$ for all $n$. Now, as $x_{n+1} \in f x_{n}$ for all $n \in$ $\mathbb{N}$, by using (15) and (18), it follows that

$$
\begin{aligned}
p\left(x_{n}, x_{n+1}\right) \leq & H_{p}\left(f x_{n-1}, f x_{n}\right)+\epsilon^{n} \\
\leq & a_{1} p\left(x_{n-1}, x_{n}\right)+a_{2} p\left(x_{n-1}, f x_{n-1}\right) \\
& +a_{3} p\left(x_{n}, f x_{n}\right)+a_{4} p\left(x_{n-1}, f x_{n}\right) \\
& +a_{5} p\left(x_{n}, f x_{n-1}\right)+\epsilon^{n} \\
\leq & a_{1} p\left(x_{n-1}, x_{n}\right)+a_{2} p\left(x_{n-1}, x_{n}\right) \\
& +a_{3} p\left(x_{n}, x_{n+1}\right)+a_{4} p\left(x_{n-1}, x_{n+1}\right) \\
& +a_{5} p\left(x_{n}, x_{n}\right)+\epsilon^{n} \\
\leq & a_{1} p\left(x_{n-1}, x_{n}\right)+a_{2} p\left(x_{n-1}, x_{n}\right) \\
& +a_{3} p\left(x_{n}, x_{n+1}\right)+a_{4} p\left(x_{n-1}, x_{n}\right) \\
& +a_{4} p\left(x_{n}, x_{n+1}\right)-a_{4} p\left(x_{n}, x_{n}\right) \\
& +a_{5} p\left(x_{n}, x_{n}\right)+\epsilon^{n},
\end{aligned}
$$

for all $n \in \mathbb{N}$; that is

$$
\begin{aligned}
p\left(x_{n}, x_{n+1}\right) \leq & \left(a_{1}+a_{2}+a_{4}\right) p\left(x_{n-1}, x_{n}\right)+\left(a_{3}+a_{4}\right) \\
& \times p\left(x_{n}, x_{n+1}\right)+\left(a_{5}+a_{4}\right) p\left(x_{n}, x_{n}\right)+\epsilon^{n}
\end{aligned}
$$

for all $n \in \mathbb{N}$. Again using symmetry of $p$ and $H_{p}$ and similar process as above with interchanging the roles of $x_{n}$ and $x_{n+1}$, we obtain

$$
\begin{aligned}
p\left(x_{n}, x_{n+1}\right) \leq & \left(a_{1}+a_{3}+a_{5}\right) p\left(x_{n-1}, x_{n}\right)+\left(a_{2}+a_{5}\right) \\
& \times p\left(x_{n}, x_{n+1}\right)+\left(a_{4}-a_{5}\right) p\left(x_{n}, x_{n}\right)+\epsilon^{n},
\end{aligned}
$$

for all $n \in \mathbb{N}$. Thus, from (20) and (21), we deduce that

$$
\begin{aligned}
2 p\left(x_{n}, x_{n+1}\right) \leq & \left(2 a_{1}+a_{2}+a_{3}+a_{4}+a_{5}\right) p\left(x_{n-1}, x_{n}\right) \\
& +\left(a_{2}+a_{3}+a_{4}+a_{5}\right) p\left(x_{n}, x_{n+1}\right)+2 \epsilon^{n} ;
\end{aligned}
$$

that is

$$
\begin{aligned}
p\left(x_{n}, x_{n+1}\right) \leq & \frac{2 a_{1}+a_{2}+a_{3}+a_{4}+a_{5}}{2-\left(a_{2}+a_{3}+a_{4}+a_{5}\right)} p\left(x_{n-1}, x_{n}\right) \\
& +\frac{2 \epsilon^{n}}{2-\left(a_{2}+a_{3}+a_{4}+a_{5}\right)} .
\end{aligned}
$$

Since $\sum_{i=1}^{5} a_{i}<1$ and $\epsilon>0$ is arbitrary, choosing $\epsilon=\left(2 a_{1}+\right.$ $\left.a_{2}+a_{3}+a_{4}+a_{5}\right) /\left(2-\left(a_{2}+a_{3}+a_{4}+a_{5}\right)\right)$, we obtain

$$
p\left(x_{n}, x_{n+1}\right) \leq \epsilon p\left(x_{n-1}, x_{n}\right)+\frac{2 \epsilon^{n}}{1+a_{1}} \quad \forall n \in \mathbb{N} \text {. }
$$

From successive application of (24), it follows that

$$
\begin{aligned}
p\left(x_{n}, x_{n+1}\right) & \leq \epsilon\left[\epsilon p\left(x_{n-2}, x_{n-1}\right)+\frac{2 \epsilon^{n-1}}{1+a_{1}}\right]+\frac{2 \epsilon^{n}}{1+a_{1}} \\
& =\epsilon^{2} p\left(x_{n-2}, x_{n-1}\right)+\frac{4 \epsilon^{n}}{1+a_{1}} \\
& \leq \epsilon^{2}\left[\epsilon p\left(x_{n-3}, x_{n-2}\right)+\frac{2 \epsilon^{n-2}}{1+a_{1}}\right]+\frac{4 \epsilon^{n}}{1+a_{1}} \\
& =\epsilon^{3} p\left(x_{n-3}, x_{n-2}\right)+\frac{6 \epsilon^{n}}{1+a_{1}} \\
& \vdots \\
& \leq \epsilon^{n} p\left(x_{0}, x_{1}\right)+\frac{2 n \epsilon^{n}}{1+a_{1}} .
\end{aligned}
$$

Note that $\epsilon<1$; therefore $\sum_{n=0}^{\infty} \epsilon^{n}<\infty$ and $\sum_{n=0}^{\infty} n \epsilon^{n}<\infty$. Consequently, we have

$$
\sum_{n=0}^{\infty} p\left(x_{n}, x_{n+1}\right) \leq p\left(x_{0}, x_{1}\right) \sum_{n=0}^{\infty} \epsilon^{n}+\frac{2}{1+a_{1}} \sum_{n=0}^{\infty} n \epsilon^{n}<\infty .
$$

Therefore $\lim _{n, m \rightarrow \infty} p\left(x_{n}, x_{m}\right)=0$ for all $n, m \in \mathbb{N}$. Thus $\left\{x_{n}\right\}$ is a 0 -Cauchy sequence in $X$. Since $(X, p)$ is 0 -complete, there exists $u \in X$ such that

$$
\lim _{n \rightarrow \infty} p\left(x_{n}, u\right)=\lim _{n, m \rightarrow \infty} p\left(x_{n}, x_{m}\right)=p(u, u)=0 .
$$

We will show that $u \in f u$; that is $u$ is a fixed point of $f$. 
Since $x_{n+1} \in f x_{n}$ for all $n \in \mathbb{N}$, we obtain that

$$
\begin{aligned}
p(u, f u) & \leq p\left(u, x_{n+1}\right)+p\left(x_{n+1}, f u\right) \\
& \leq p\left(u, x_{n+1}\right)+H_{p}\left(f x_{n}, f u\right) .
\end{aligned}
$$

By using (15) we get

$$
\begin{aligned}
p(u, f u) \leq & p\left(u, x_{n+1}\right)+a_{1} p\left(x_{n}, u\right)+a_{2} p\left(x_{n}, f x_{n}\right) \\
& +a_{3} p(u, f u)+a_{4} p\left(x_{n}, f u\right)+a_{5} p\left(u, f x_{n}\right) \\
\leq & p\left(u, x_{n+1}\right)+a_{1} p\left(x_{n}, u\right)+a_{2} p\left(x_{n}, x_{n+1}\right) \\
& +a_{3} p(u, f u)+a_{4} p\left(x_{n}, f u\right)+a_{5} p\left(u, x_{n+1}\right) \\
\leq & p\left(u, x_{n+1}\right)+a_{1} p\left(x_{n}, u\right)+a_{2} p\left(x_{n}, u\right) \\
& +a_{2} p\left(x_{n+1}, u\right)-a_{2} p(u, u) \\
& +a_{3} p(u, f u)+a_{4} p\left(x_{n}, u\right)+a_{4} p(u, f u) \\
& -a_{4} p(u, u)+a_{5} p\left(x_{n+1}, u\right) \\
= & \left(a_{1}+a_{2}+a_{4}\right) p\left(x_{n}, u\right) \\
& +\left(1+a_{2}+a_{5}\right) p\left(x_{n+1}, u\right) \\
& -\left(a_{2}+a_{4}\right) p(u, u)+\left(a_{3}+a_{4}\right) p(u, f u)
\end{aligned}
$$

that is

$$
\begin{aligned}
p(u, f u) \leq & \frac{a_{1}+a_{2}+a_{4}}{1-\left(a_{3}+a_{4}\right)} p\left(x_{n}, u\right) \\
& +\frac{1+a_{2}+a_{5}}{1-\left(a_{3}+a_{4}\right)} p\left(x_{n+1}, u\right) .
\end{aligned}
$$

By using (27), from the above inequality, it follows that $p(u$, $f u)=0=p(u, u)$. Therefore by Lemma 7, we have $u \in f u$. Thus $u$ is a fixed point of $f$.

Remark 16. In [7], authors used a Banach type contractive condition on $f$. In the above theorem we use a more general Hardy-Rogers type contractive condition on $f$. Also, the completeness of space is replaced by 0 -completeness, which is more general than completeness. Our method for the proof of the main result is different from the method used in [7]. Also, if $p$ is replaced with a metric $d$ on $X$, we obtain Hardy-Rogers theorem for usual metric spaces.

With suitable values of control constants $a_{1}, a_{2}, a_{3}, a_{4}, a_{5}$, the following corollaries are obtained.

Corollary 17 (see [7], Banach type). Let $(X, p)$ be a 0-complete partial metric space and $f: X \rightarrow C B^{p}(X)$ be a mapping such that

$$
H_{p}(f x, f y) \leq \lambda p(x, y)
$$

for all $x, y \in X$, where $\lambda \in[0,1)$. Then $f$ has a fixed point in $X$.
Corollary 18 (Kannan type). Let $(X, p)$ be a 0-complete partial metric space and $f: X \rightarrow C B^{p}(X)$ be a mapping such that

$$
H_{p}(f x, f y) \leq \lambda[p(x, f x)+p(y, f y)]
$$

for all $x, y \in X$, where $\lambda \in[0,1 / 2)$. Then $f$ has a fixed point in $X$.

Corollary 19 (Reich type). Let $(X, p)$ be a 0-complete partial metric space and $f: X \rightarrow C B^{p}(X)$ be a mapping such that

$$
H_{p}(f x, f y) \leq a p(x, y)+b p(x, f x)+c p(y, f y)
$$

for all $x, y \in X$, where $a, b, c$ are nonnegative constants such that $a+b+c<1$. Then $f$ has a fixed point in $X$.

Corollary 20 (Chatterjea type). Let $(X, p)$ be a 0-complete partial metric space and $f: X \rightarrow C B^{p}(X)$ be a mapping such that

$$
H_{p}(f x, f y) \leq \lambda[p(x, f y)+p(y, f x)]
$$

for all $x, y \in X$, where $\lambda \in[0,1 / 2)$. Then $f$ has a fixed point in $X$.

Corollary 21 (Ćirić type). Let $(X, p)$ be a 0 -complete partial metric space and $f: X \rightarrow C B^{p}(X)$ be a mapping such that

$$
\begin{aligned}
H_{p}(f x, f y) \leq & a p(x, y)+b p(x, f x)+c p(y, f y) \\
& +e[p(x, f y)+p(y, f x)],
\end{aligned}
$$

for all $x, y \in X$, where $a, b, c$, e are nonnegative constants such that $a+b+c+2 e<1$. Then $f$ has a fixed point in $X$.

The following examples illustrate the cases when the results of Aydi et al. [7] and Nadler Jr. [3] are not applicable, while the new results are applicable.

Example 22. Let $X=\{0,1,2\}$ be endowed with the partial metric $p: X \times X \rightarrow \mathbb{R}^{+}$defined by

$$
p(x, y)= \begin{cases}0, & \text { if } x=y=2 \\ |x-y|+\max \{x, y\}, & \text { otherwise. }\end{cases}
$$

Obviously, $p$ is not a metric on $X$ and $(X, p)$ is a 0 -complete partial metric space. Note that $\left(X, p_{1}\right)$ where

$$
p_{1}(x, y)= \begin{cases}0, & \text { if } x=y=2 ; \\ \max \{x, y\}, & \text { otherwise }\end{cases}
$$

is a partial metric space and $(X, d)$ where $d$ is the usual metric on $X$ is a metric space; also $p(x, y)=d(x, y)+p_{1}(x, y)$ for all $x, y \in X$. Therefore, in view of Corollary $14,\left(X, \tau_{p}\right)$ is a $T_{1}$ space and hence all subsets of $X$ are closed (also bounded).

Define $f: X \rightarrow C B^{p}(X)$ by

$$
f 0=\{0\}, \quad f 1=\{0\}, \quad f 2=\{0,1\} .
$$


We will show that $f$ is not a contraction in the sense of Aydi et al. [7] and Nadler Jr. [3]. Note that, for $x=y=2$, we have

$$
\begin{aligned}
H_{p}(f x, f y) & =H_{p}(\{0,1\},\{0,1\}) \\
& =\max _{x \in\{0,1\}} p(x, x)=\max \{0,1\}=1,
\end{aligned}
$$

and $p(x, y)=p(2,2)=0$. Therefore, there is no real $\lambda$ such that $H_{p}(f x, f y) \leq \lambda p(x, y)$ with $\lambda \in(0,1)$. Thus, $f$ is not a contraction in the sense of Aydi et al. [7] and we cannot conclude the existence of fixed point of $f$ with the assumptions of [7].

Note that $f$ is not a set-valued contraction in usual metric space $(X, d)$. Indeed, if $H_{d}$ represents the Hausdorff metric induced by $d$, then for $x=1$ and $y=2$ we have $H_{d}(f x, f y)=$ 1 and $d(x, y)=1$; therefore there is no real $\lambda \in[0,1)$ such that $H_{d}(f x, f y) \leq \lambda d(x, y)$. Therefore, the result of Nadler Jr. [3] is not applicable on $f$.

Again, if $H_{p^{s}}$ represents the Hausdorff metric induced by $p^{s}$, where $p^{s}$ (induced by $p$ ) is given by

$$
p^{s}(x, y)=2|x-y|+2 \max \{x, y\}-x-y, \quad \forall x, y \in X,
$$

then for $x=1$ and $y=2$ we have $H_{p^{s}}(f x, f y)=3$ and $p^{s}$ $(x, y)=3$. Also in this case, there is no real $\lambda \in[0,1)$ such that $H_{p^{s}}(x, y) \leq \lambda p^{s}(x, y)$. Therefore, again the result of Nadler Jr. is not applicable on $f$.

Now by careful calculations, one can see that $f$ satisfies all the conditions of Corollary 21, with $a=1 / 2, b=c=e=$ $1 / 12$. Thus, there must be at least one fixed point of $f$; here $x=0$ is a fixed point of $f$.

Example 23. Let $X=\mathbb{Q} \cap[0,1]$ be endowed with the partial metric $p: X \times X \rightarrow \mathbb{R}^{+}$defined by

$$
p(x, y)=|x-y|+\max \{x, y\}, \quad \forall x, y \in X .
$$

Then $(X, p)$ is a partial metric space. Note that $p(x, y)=d(x$, $y)+p_{1}(x, y)$ for all $x, y \in X$, where $d$ is the usual metric on $X$ and $p_{1}: X \times X \rightarrow \mathbb{R}^{+}$, defined by $p_{1}(x, y)=$ $\max \{x, y\}$, for all $x, y \in X$, is a partial metric on $X$. In view of Corollary $14,\left(X, \tau_{p}\right)$ is a $T_{1}$ space. Also, $(X, p)$ is 0 -complete but not complete, because the induced metric space $\left(X, p^{s}\right)$, where $p^{s}(x, y)=2 d(x, y)+2 p_{1}(x, y)-x-y$ for all $x, y \in X$, is not complete. Define $f: X \rightarrow C B^{p}(X)$ by

$$
f x= \begin{cases}\{0\}, & \text { if } x=1 \\ \left\{0, \frac{x}{4}\right\}, & \text { otherwise. }\end{cases}
$$

Then, $f$ satisfies all the conditions of Corollary 17 with $a \in$ $[1 / 2,1)$ and has a fixed point, $x=0$. Note that the result of Aydi et al. [7] is not applicable (since the partial metric space $(X, p)$ is not complete).

In Theorem 15, the fixed point of $f$ is a limit of sequence $\left\{x_{n}\right\}$, and, due to convergence in 0-complete space, this fixed point has zero self-distance. The following remark is helpful in proving the next homotopy result; it shows that, if $f$ has a fixed point in $X$, then its self-distance must be zero.
Remark 24. Let $(X, p)$ be a partial metric space and $f: X \rightarrow$ $C B^{p}(X)$ be a mapping satisfying (15). If $u \in f u$ for some $u \in$ $X$, then $p(x, x)=0$ for all $x \in f u$, and hence $H_{p}(f u, f u)=0$.

Proof. Let $u \in f u \in C B^{p}(X)$, then, by Lemma 7, $p(u, f u)=$ $p(u, u)$ and $H_{p}(f u, f u)=\delta_{p}(f u, f u)=\sup _{x \in f u} p(x, x)$. Suppose $p(u, u)>0$; then from (15) we have

$$
\begin{aligned}
& H_{p}(f u, f u) \leq a_{1} p(u, u)+a_{2} p(u, f u) \\
&+a_{3} p(u, f u)+a_{4} p(u, f u)+a_{5} p(u, f u), \\
& \sup _{x \in f u} p(x, x) \leq\left(a_{1}+a_{2}+a_{3}+a_{4}+a_{5}\right) p(u, u), \\
& \sup _{x \in f u} p(x, x)<p(u, u) .
\end{aligned}
$$

Since $u \in f u$, the above inequality yields a contradiction and so the result holds true.

\section{Application to Homotopy Results}

In this section, as an application of our main result, we derive a homotopy result.

Let $\eta:[a, b]^{2} \rightarrow \mathbb{R}^{+}$be a function such that one of the following conditions holds:

$\left(\eta_{1}\right)$ for all $r, s, t, u, v \in[a, b]$, we have $\eta(t, r) \leq \eta(t, s)+$ $\eta(r, s)$ and $\eta(u, v) \rightarrow 0$ if $u \rightarrow v$;

$\left(\eta_{2}\right)$ for all $s, t \in[a, b]$ and some $L>0$, we have $\eta(t, s) \leq$ $L|t-s|$.

Theorem 25. Let $(X, p)$ be a 0 -complete partial metric space, $F$ be a closed subset of $X$, and $U$ be an open subset of $X$ with $U \subset F$. Let $a, b \in \mathbb{R}$ and $f: F \times[a, b] \rightarrow C B^{p}(X)$ be an operator such that the following conditions hold:

(a) $x \notin f(x, t)$ for each $x \in F \backslash U$ and each $t \in[a, b]$;

(b) there exist nonnegative constants $a_{1}, a_{2}, a_{3}, a_{4}, a_{5}$ such that $\sum_{i=1}^{5} a_{i}<1$ and, for all $x, y \in F$ and each $t \in[a, b]$, we have

$$
\begin{aligned}
H_{p}( & f(x, t), f(y, t)) \\
\leq & a_{1} p(x, y)+a_{2} p(x, f(x, t))+a_{3} p(y, f(y, t)) \\
& +a_{4} p(x, f(y, t))+a_{5} p(y, f(x, t))
\end{aligned}
$$

(c) there exists $M>0$ such that

$$
\begin{array}{r}
H_{p}\left(f\left(x, t_{1}\right), f\left(x, t_{2}\right)\right) \leq M \eta\left(t_{1}, t_{2}\right) \\
\forall t_{1}, t_{2} \in[a, b] \text { and each } x \in F ;
\end{array}
$$

(d) if $x \in f(x, t)$, then $f(x, t)=\{x\}$.

If $f\left(\cdot, t_{1}\right)$ has a fixed point in $F$ for at least one $t_{1} \in[a, b]$, then $f(\cdot, t)$ has a fixed point in $U$ for all $t \in[a, b]$. Furthermore, for any fixed $t \in[a, b]$, the fixed point of $f(\cdot, t)$ is unique. 
Proof. Define

$$
\mathbb{Q}:=\{t \in[a, b]: x \in f(x, t) \text { for some } x \in U\} .
$$

As $f\left(\cdot, t_{1}\right)$ has a fixed point in $F$ for at least one $t_{1} \in[a, b]$; that is there exists $x \in F$ such that $x \in f\left(x, t_{1}\right)$ for at least one $t_{1} \in$ $[a, b]$, and (a) holds and therefore $Q \neq \emptyset$. We will show that $\mathbb{Q}$ is both open and closed in $[a, b]$ and so, by connectedness of $[a, b], Q=[a, b]$.

(I) $Q$ is closed. Let $\left\{t_{n}\right\}$ be a sequence in $\mathbb{Q}$ and $t_{n} \rightarrow s \in$ $[a, b]$ as $n \rightarrow \infty$. We must show that $s \in \mathbb{Q}$. Since $t_{n} \in \mathbb{Q}$ for all $n \in \mathbb{N}$, there exists $x_{n} \in U$ with $x_{n} \in f\left(x_{n}, t_{n}\right)$ for all $n \in \mathbb{N}$. For $n, m \in \mathbb{N}$ with $m>n$, using (b), (c), and Remark 24, we obtain

$$
\begin{aligned}
& H_{p}\left(f\left(x_{n}, t_{m}\right), f\left(x_{m}, t_{m}\right)\right) \\
& \leq a_{1} p\left(x_{n}, x_{m}\right)+a_{2} p\left(x_{n}, f\left(x_{n}, t_{m}\right)\right) \\
&+a_{3} p\left(x_{m}, f\left(x_{m}, t_{m}\right)\right)+a_{4} p\left(x_{n}, f\left(x_{m}, t_{m}\right)\right) \\
&+a_{5} p\left(x_{m}, f\left(x_{n}, t_{m}\right)\right) \\
& \leq a_{1} p\left(x_{n}, x_{m}\right)+a_{2} H_{p}\left(f\left(x_{n}, t_{n}\right), f\left(x_{n}, t_{m}\right)\right) \\
&+a_{4} p\left(x_{n}, x_{m}\right)+a_{5} H_{p}\left(f\left(x_{m}, t_{m}\right), f\left(x_{n}, t_{m}\right)\right) ;
\end{aligned}
$$

that is

$$
\begin{aligned}
& \left(1-a_{5}\right) H_{p}\left(f\left(x_{n}, t_{m}\right), f\left(x_{m}, t_{m}\right)\right) \\
& \quad \leq\left(a_{1}+a_{4}\right) p\left(x_{n}, x_{m}\right)+a_{2} M \eta\left(t_{n}, t_{m}\right) .
\end{aligned}
$$

Therefore, using (48), (d), and (h3) of Proposition 9, we obtain

$$
\begin{aligned}
\left(1-a_{5}\right) p\left(x_{n}, x_{m}\right)= & \left(1-a_{5}\right) H_{p}\left(f\left(x_{n}, t_{n}\right), f\left(x_{m}, t_{m}\right)\right) \\
\leq & \left(1-a_{5}\right) \\
\times & {\left[H_{p}\left(f\left(x_{n}, t_{n}\right), f\left(x_{n}, t_{m}\right)\right)\right.} \\
& +H_{p}\left(f\left(x_{n}, t_{m}\right), f\left(x_{m}, t_{m}\right)\right) \\
& \left.\quad-\inf _{x \in f\left(x_{n}, t_{m}\right)} p(x, x)\right] \\
\leq & \left(1-a_{5}\right) H_{p}\left(f\left(x_{n}, t_{n}\right), f\left(x_{n}, t_{m}\right)\right) \\
& +\left(1-a_{5}\right) H_{p}\left(f\left(x_{n}, t_{m}\right), f\left(x_{m}, t_{m}\right)\right) \\
\leq & \left(1-a_{5}\right) M \eta\left(t_{n}, t_{m}\right) \\
& +\left(a_{1}+a_{4}\right) p\left(x_{n}, x_{m}\right)+a_{2} M \eta\left(t_{n}, t_{m}\right) \\
= & \left(1+a_{2}-a_{5}\right) M \eta\left(t_{n}, t_{m}\right) \\
& +\left(a_{1}+a_{4}\right) p\left(x_{n}, x_{m}\right) ;
\end{aligned}
$$

that is

$$
p\left(x_{n}, x_{m}\right) \leq \frac{\left(1+a_{2}-a_{5}\right) M}{1-\left(a_{1}+a_{4}+a_{5}\right)} \eta\left(t_{n}, t_{m}\right) .
$$

Using the properties of $\eta$ and the fact that $\lim _{n \rightarrow \infty} t_{n}=s$, we get easily that $\eta\left(t_{n}, t_{m}\right) \rightarrow 0$ as $n \rightarrow \infty$. Since $\sum_{i=1}^{5} a_{i}<1$, from (50), we deduce that

$$
\lim _{n \rightarrow \infty} p\left(x_{n}, x_{m}\right)=0 .
$$

Thus $\left\{x_{n}\right\}$ is a 0 -Cauchy sequence in $F,(X, p)$ is 0 -complete, and $F$ is closed; therefore there exists $u \in F$ such that

$$
\lim _{n \rightarrow \infty} p\left(x_{n}, u\right)=p(u, u)=0 .
$$

For any $n \in \mathbb{N}$, we have

$$
p\left(x_{n}, f\left(x_{n}, s\right)\right) \leq H_{p}\left(f\left(x_{n}, t_{n}\right), f\left(x_{n}, s\right)\right) \leq M \eta\left(t_{n}, s\right),
$$

and, hence, using the properties of $\eta, \lim _{n \rightarrow \infty} p\left(x_{n}, f\left(x_{n}\right.\right.$, $s))=0$. Also

$$
\begin{aligned}
p\left(u, f\left(x_{n}, s\right)\right) & \leq p\left(u, x_{n}\right)+p\left(x_{n}, f\left(x_{n}, s\right)\right) \\
& \leq p\left(u, x_{n}\right)+H_{p}\left(f\left(x_{n}, t_{n}\right), f\left(x_{n}, s\right)\right) \\
& \leq p\left(u, x_{n}\right)+M \eta\left(t_{n}, s\right) .
\end{aligned}
$$

Using the properties of $\eta$, the above inequality implies $\lim _{n \rightarrow \infty} p\left(u, f\left(x_{n}, s\right)\right)=0$. Now

$$
\begin{aligned}
p\left(x_{n}, f(u, s)\right) \leq & H_{p}\left(f\left(x_{n}, t_{n}\right), f(u, s)\right) \\
\leq & H_{p}\left(f\left(x_{n}, t_{n}\right), f\left(x_{n}, s\right)\right) \\
& +H_{p}\left(f\left(x_{n}, s\right), f(u, s)\right) \\
& -\inf _{x \in f\left(x_{n}, s\right)} p(x, x) \\
\leq & M \eta\left(t_{n}, s\right)+a_{1} p\left(x_{n}, u\right) \\
& +a_{2} p\left(x_{n}, f\left(x_{n}, s\right)\right)+a_{3} p(u, f(u, s)) \\
& +a_{4} p\left(x_{n}, f(u, s)\right)+a_{5} p\left(u, f\left(x_{n}, s\right)\right),
\end{aligned}
$$

or equivalently

$$
\begin{aligned}
\left(1-a_{4}\right) p\left(x_{n}, f(u, s)\right) \leq & M \eta\left(t_{n}, s\right)+a_{1} p\left(x_{n}, u\right) \\
& +a_{2} p\left(x_{n}, f\left(x_{n}, s\right)\right) \\
& +a_{3} p(u, f(u, s)) \\
& +a_{5} p\left(u, f\left(x_{n}, s\right)\right) .
\end{aligned}
$$


Using the above inequality we obtain

$$
\begin{aligned}
\left(1-a_{4}\right) p(u, f(u, s)) \leq & \left(1-a_{4}\right)\left[p\left(u, x_{n}\right)+p\left(x_{n}, f(u, s)\right)\right] \\
= & \left(1-a_{4}\right) p\left(u, x_{n}\right)+M \eta\left(t_{n}, s\right) \\
& +a_{1} p\left(x_{n}, u\right)+a_{2} p\left(x_{n}, f\left(x_{n}, s\right)\right) \\
& +a_{3} p(u, f(u, s)) \\
& +a_{5} p\left(u, f\left(x_{n}, s\right)\right)
\end{aligned}
$$

that is

$$
\begin{aligned}
\left(1-a_{3}-a_{4}\right) p(u, f(u, s)) & \\
\leq & \left(1-a_{4}\right) p\left(u, x_{n}\right)+M \eta\left(t_{n}, s\right)+a_{1} p\left(x_{n}, u\right) \\
& +a_{2} p\left(x_{n}, f\left(x_{n}, s\right)\right)+a_{5} p\left(u, f\left(x_{n}, s\right)\right) .
\end{aligned}
$$

Since $p\left(x_{n}, f\left(x_{n}, s\right)\right) \rightarrow 0, p\left(u, f\left(x_{n}, s\right)\right) \rightarrow 0$ as $n \rightarrow \infty$, $1-a_{3}-a_{4}>0$ and, using the properties of $\eta$, it follows from the above inequality that $p(u, f(u, s))=0$. Therefore $u \in f(u, s)$, and from (a) we deduce that $u \in U$. Thus $s \in \mathbb{Q}$ and hence $\mathbb{Q}$ is closed in $[a, b]$.

(II) $\mathbb{Q}$ is open. Let $t_{0} \in \mathbb{Q}$ and $x_{0} \in U$ with $x_{0} \in f\left(x_{0}, t_{0}\right)$. Note that, for this $t_{0}$, Remark 24 is applicable and so

$$
p\left(x_{0}, x_{0}\right)=0 \text {. }
$$

Since $U$ is open, there exists $r>0$ such that $B_{p}\left(x_{0}, r\right) \subset U$. Suppose $\epsilon=((1-k) / M) r>0$, where $k=\left(2 a_{1}+a_{2}+a_{3}+a_{4}+\right.$ $\left.a_{5}\right) /\left(2-a_{2}-a_{3}-a_{4}-a_{5}\right)<1$. By the definition of $\eta$ we can choose $\delta>0$ such that $\eta\left(t, t_{0}\right)<\epsilon$ for all $t \in\left(t_{0}-\delta, t_{0}+\delta\right)$.

Let $t \in\left(t_{0}-\delta, t_{0}+\delta\right)$; then, for all $x \in \overline{B_{p}\left(x_{0}, r\right)}=\{x \in$ $\left.X: p\left(x, x_{0}\right) \leq r+p\left(x_{0}, x_{0}\right)\right\}=\left\{x \in X: p\left(x, x_{0}\right) \leq r\right\}$ (as $\left.p\left(x_{0}, x_{0}\right)=0\right)$, we shall show that $f(x, t) \subset \overline{B_{p}\left(x_{0}, r\right)}$, and so $f: \overline{B_{p}\left(x_{0}, r\right)} \rightarrow C B^{p}\left(\overline{B_{p}\left(x_{0}, r\right)}\right)$. Let $x \in \overline{B_{p}\left(x_{0}, r\right)}$; then we have

$$
\begin{aligned}
H_{p}( & \left.f\left(x, t_{0}\right), f\left(x_{0}, t_{0}\right)\right) \\
\leq & a_{1} p\left(x, x_{0}\right)+a_{2} p\left(x, f\left(x, t_{0}\right)\right)+a_{3} p\left(x_{0}, f\left(x_{0}, t_{0}\right)\right) \\
& +a_{4} p\left(x, f\left(x_{0}, t_{0}\right)\right)+a_{5} p\left(x_{0}, f\left(x, t_{0}\right)\right) \\
\leq & a_{1} p\left(x, x_{0}\right)+a_{2}\left[p\left(x, x_{0}\right)+p\left(x_{0}, f\left(x, t_{0}\right)\right)\right] \\
& +a_{3} p\left(x_{0}, x_{0}\right)+a_{4} p\left(x, x_{0}\right)+a_{5} p\left(x_{0}, f\left(x, t_{0}\right)\right) .
\end{aligned}
$$

Since $p\left(x_{0}, f\left(x, t_{0}\right)\right) \leq H_{p}\left(f\left(x_{0}, t_{0}\right), f\left(x, t_{0}\right)\right)$, we obtain

$$
\begin{aligned}
H_{p}( & \left.f\left(x, t_{0}\right), f\left(x_{0}, t_{0}\right)\right) \\
\leq & \left(a_{1}+a_{2}+a_{4}\right) p\left(x, x_{0}\right) \\
\quad & +\left(a_{2}+a_{5}\right) H_{p}\left(f\left(x_{0}, t_{0}\right), f\left(x, \mathrm{t}_{0}\right)\right) .
\end{aligned}
$$

Again, since $p$ and $H_{p}$ are symmetric, applying a similar process as above, we get

$$
\begin{aligned}
& H_{p}(\left.f\left(x_{0}, t_{0}\right), f\left(x, t_{0}\right)\right) \\
& \leq\left(a_{1}+a_{3}+a_{5}\right) p\left(x, x_{0}\right) \\
& \quad+\left(a_{3}+a_{4}\right) H_{p}\left(f\left(x_{0}, t_{0}\right), f\left(x, t_{0}\right)\right) .
\end{aligned}
$$

From (61) and (62) we deduce that

$$
\begin{aligned}
H_{p}\left(f\left(x, t_{0}\right), f\left(x_{0}, t_{0}\right)\right) & \leq \frac{2 a_{1}+a_{2}+a_{3}+a_{4}+a_{5}}{2-a_{2}-a_{3}-a_{4}-a_{5}} p\left(x, x_{0}\right) \\
& =k p\left(x, x_{0}\right) .
\end{aligned}
$$

Suppose $y \in f(x, t)$; then using (63) we obtain

$$
\begin{aligned}
p\left(y, x_{0}\right)= & p\left(y, f\left(x_{0}, t_{0}\right)\right) \leq H_{p}\left(f(x, t), f\left(x_{0}, t_{0}\right)\right) \\
\leq & H_{p}\left(f(x, t), f\left(x, t_{0}\right)\right) \\
& +H_{p}\left(f\left(x, t_{0}\right), f\left(x_{0}, t_{0}\right)\right)-\inf _{z \in f\left(x, t_{0}\right)} p(z, z) \\
\leq & M \eta\left(t, t_{0}\right)+k p\left(x, x_{0}\right)<M \epsilon+k r \\
\leq & (1-k) r+k r=r .
\end{aligned}
$$

Therefore $y \in \overline{B_{p}\left(x_{0}, r\right)}$ and so, for each fixed $t \in\left(t_{0}-\delta\right.$, $\left.t_{0}+\delta\right)$, we have $f(x, t) \subset \overline{B_{p}\left(x_{0}, r\right)}$. Thus $f(\cdot, t): \overline{B_{p}\left(x_{0}, r\right)} \rightarrow$ $C B^{p}\left(\overline{B_{p}\left(x_{0}, r\right)}\right)$ and $f(\cdot, t)$ satisfies all the hypotheses of Theorem 15 and has a fixed point in $\overline{B_{p}\left(x_{0}, r\right)} \subset F$. By (a), this fixed point must be in $U$; therefore $\left(t_{0}-\delta, t_{0}+\delta\right) \subset \mathbb{Q}$ and hence $\mathbb{Q}$ is open. Thus $Q=[a, b]$ and $f(\cdot, t)$ has a fixed point in $U$ for all $t \in[a, b]$.

For uniqueness, fixed $t \in[a, b]$; then there exists $x \in C$ such that $x \in f(x, t)$. If $y$ is another fixed point of $f(\cdot, t)$, then from $(\mathrm{d})$ we have

$$
\begin{aligned}
p(x, y)= & H_{p}(f(x, t), f(y, t)) \\
\leq & a_{1} p(x, y)+a_{2} p(x, f(x, t)) \\
& +a_{3} p(y, f(y, t))+a_{4} p(x, f(y, t)) \\
& +a_{5} p(y, f(x, t)) \\
= & a_{1} p(x, y)+a_{2} p(x, x)+a_{3} p(y, y) \\
& +a_{4} p(x, y)+a_{5} p(y, x) \\
\leq & \left(a_{1}+a_{2}+a_{3}+a_{4}+a_{5}\right) p(x, y) \\
< & p(x, y),
\end{aligned}
$$

which is a contradiction. Therefore, for any fixed $t \in[a, b]$, the fixed point of $f(\cdot, t)$ is unique.

Remark 26. In Theorem 25, to prove the existence of the fixed point of $f(\cdot, t)$, it is assumed that $f\left(\cdot, t_{1}\right)$ has a fixed point 
in the closed set $F$. Now, note that in Theorem 4.1 of [7], to prove the existence of the fixed point of $f(\cdot, 1)$, authors assumed that $f(\cdot, 0)$ has a fixed point (not necessarily in the closed set $F$ ). Since every metric space is a partial metric space with zero self-distance, therefore the homotopy result of [7] must be valid in metric spaces. Also, in the proof of Theorem 4.1 of [7] authors claimed that the set $Q:=\{t \in$ $[0,1]: x \in f(x, t)$ for some $x \in U\}$ is nonempty, but the next example shows that, if the fixed points of $f(\cdot, 0)$ are not in $F$, then it can happen that $Q=\emptyset$. Thus, Theorem 25 of this paper is a corrected, improved, and generalized version of the homotopy result of [7].

Example 27. Let $X=[0,1]$; then $(X, d)$ is a complete metric space, where $d$ is the usual metric on $X$. Let $U=(1 / 2,3 / 4)$ an $F=[1 / 2,1]$ and define $f: F \times[0,1] \rightarrow C B(X)$ by $f(x, t)=$ $\{x / 2-3 t x / 8\}$ for all $x \in X, t \in[0,1]$. If $H_{d}$ is the Hausdorff metric induced by $d$, then we have

(1) $x \notin f(x, t)$ for each $x \in F \backslash U$;

(2) for all $x, y \in F$ and each $t \in[0,1]$ we have

$H_{d}(f(x, t), f(y, t)) \leq \lambda d(x, y), \quad$ where $\lambda \in\left[\frac{1}{2}, 1\right) ;$

(3) $H_{d}(f(x, t), f(x, s)) \leq \lambda|t-s|$ for all $t, s \in[0,1]$ and each $x \in F$;

(4) if $x \in f(x, t)$, then $f(x, t)=\{x\}$.

Note that $0(\notin F)$ is a unique fixed point of $f(\cdot, t)$ in $X$, for all $t \in[0,1]$. Therefore, all the conditions of Theorem 4.1 of [7] are satisfied, but $Q:=\{t \in[0,1]: x \in f(x, t)$ for some $x \in$ $U\}=\emptyset$.

\section{Conflict of Interests}

The authors declare that there is no conflict of interests regarding the publication of this paper.

\section{Acknowledgments}

The authors gratefully acknowledge the suggestions and advice of the editor and anonymous reviewer(s). The third author is a member of the Gruppo Nazionale per l'Analisi Matematica, la Probabilità e le loro Applicazioni (GNAMPA) of the Istituto Nazionale di Alta Matematica (INdAM).

\section{References}

[1] G. E. Hardy and T. D. Rogers, "A generalization of a fixed point theorem of Reich," Canadian Mathematical Bulletin, vol. 16, pp. 201-206, 1973.

[2] B. E. Rhoades, "A comparison of various definitions of contractive mappings," Transactions of the American Mathematical Society, vol. 226, pp. 257-290, 1977.

[3] S. B. Nadler Jr., "Multi-valued contraction mappings," Pacific Journal of Mathematics, vol. 30, pp. 475-488, 1969.
[4] S. G. Matthews, "Partial metric topology," in Papers on General Topology and Applications (Flushing, NY, 1992), vol. 728 of Annals of the New York Academy of Sciences, pp. 183-197, The New York Academy of Sciences, New York, NY, USA, 1994.

[5] M. Bukatin, R. Kopperman, S. Matthews, and H. Pajoohesh, "Partial metric spaces," American Mathematical Monthly, vol. 116, no. 8, pp. 708-718, 2009.

[6] S. Romaguera, "A Kirk type characterization of completeness for partial metric spaces," Fixed Point Theory and Applications, vol. 2010, Article ID 493298, 6 pages, 2010.

[7] H. Aydi, M. Abbas, and C. Vetro, "Partial Hausdorff metric and Nadler's fixed point theorem on partial metric spaces," Topology and Its Applications, vol. 159, no. 14, pp. 3234-3242, 2012.

[8] Z. Kadelburg, H. K. Nashine, and S. Radenović, "Fixed point results under various contractive conditions in partial metric spaces," Revista de la Real Academia de Ciencias Exactas, Fisicas y Naturales. Serie A. Matematicas, vol. 107, no. 2, pp. 241-256, 2013.

[9] I. Altun, F. Sola, and H. Simsek, "Generalized contractions on partial metric spaces," Topology and Its Applications, vol. 157, no. 18, pp. 2778-2785, 2010.

[10] S. Romaguera, "Matkowski’s type theorems for generalized contractions on (ordered) partial metric spaces," Applied General Topology, vol. 12, no. 2, pp. 213-220, 2011. 


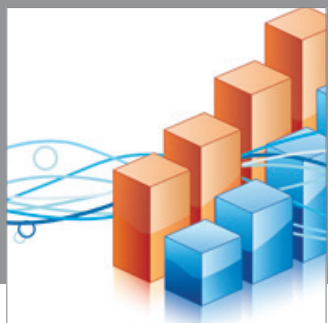

Advances in

Operations Research

mansans

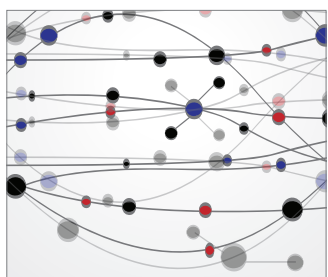

The Scientific World Journal
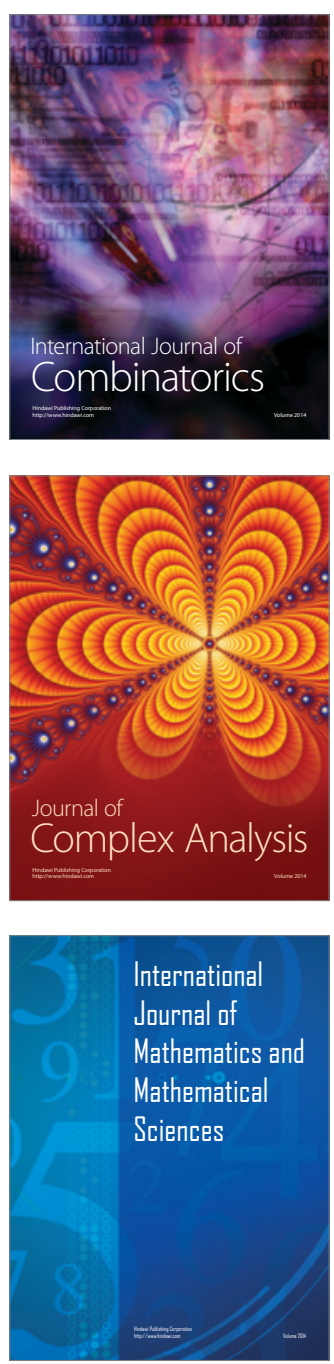
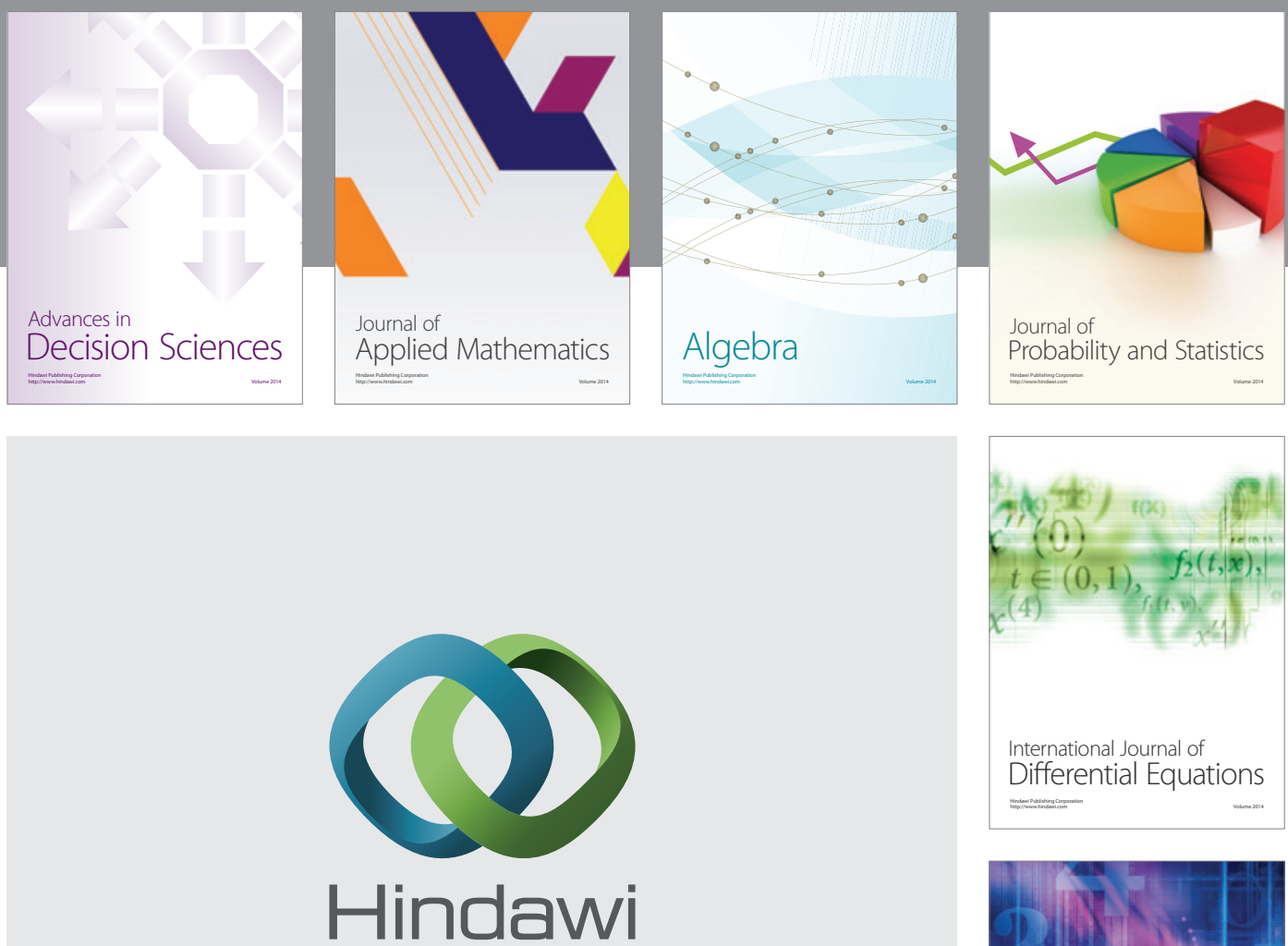

Submit your manuscripts at http://www.hindawi.com
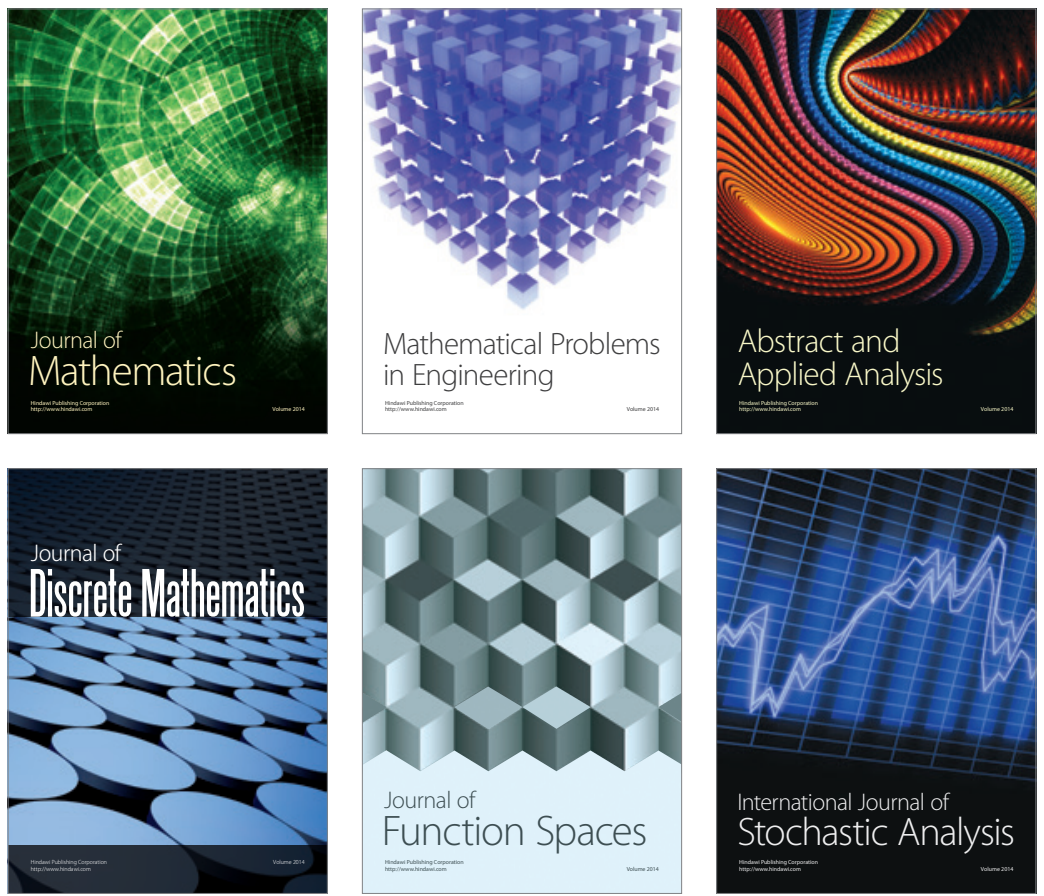

Journal of

Function Spaces

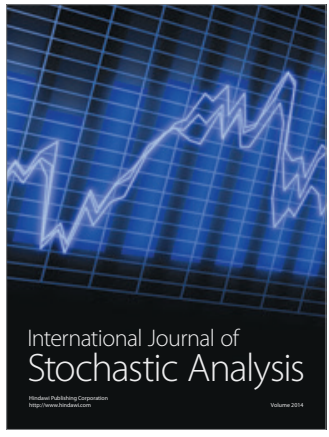

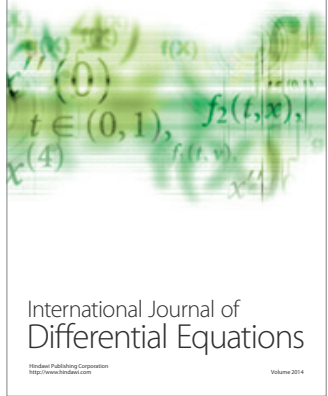
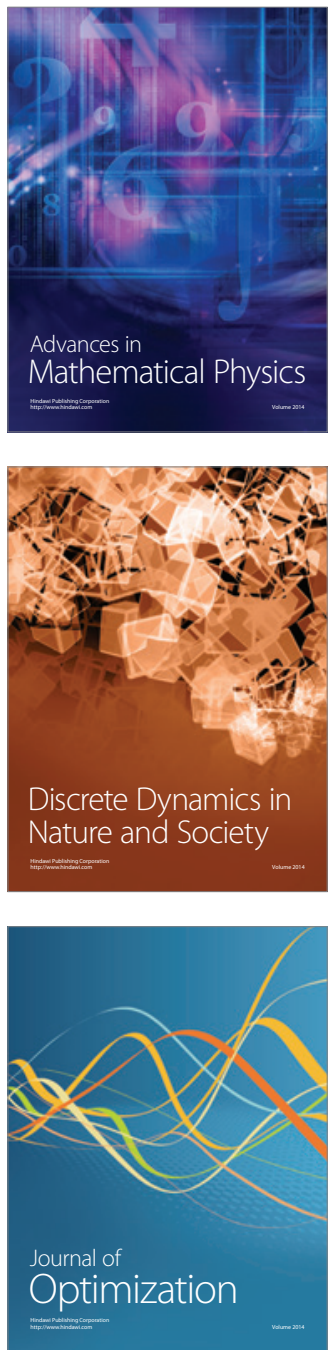